\title{
An Efficient Generic Framework for Three-Factor Authentication with Provably Secure Instantiation
}

\author{
Jiangshan Yu*, Guilin Wang, Yi Mu, Senior Member, IEEE and Wei Gao
}

\begin{abstract}
Remote authentication has been widely studied and adapted in distributed systems. The security of remote authentication mechanisms mostly relies on one of or the combination of three factors: something users know - password, something users have - smart card, and something users are - biometric characteristics.

This paper introduces an efficient generic framework for threefactor authentication. The proposed generic framework enhances the security of existing two-factor authentication schemes by upgrading them to three-factor authentication schemes, without exposing user privacy. In addition, we present a case study by upgrading a secure two-factor authentication scheme to a secure three-factor authentication scheme. Furthermore, implementation analysis, formal proof and privacy discussion are provided to show that the derived scheme is practical, secure and privacypreserving.
\end{abstract}

Index Terms-Authentication, security, privacy, password, smart card, biometrics.

\section{INTRODUCTION}

$\mathbf{T}$ HE need of user authentication is a fundamental security requirement in computer society. With wide-spread of distributed computer networks, remote user authentication has been introduced to identify a user remotely, and has been widely studied (e.g. [1], [2], [3]). In general, authentication services may require three factors, i.e., password, smart card and biometric characteristics. The authentication based on a password is called password-based authentication (e.g. Facebook login system). A system which authenticates users by using password and smart card is called two-factor authentication (e.g. HSBC Internet banking login system). In which, a client can pass authentication only if the client has correct password and the corresponding authentic smart card. The biometric-based authentication mainly employs the biometric characteristics, e.g. fingerprint, palm print, and iris.

The earliest user authentication mechanism through the Internet is based on password. The concept of password based authentication was first proposed by Lamport in 1981 [4]. Such

* Corresponding author.

Jiangshan Yu is with the Department of Computer Science, University of Birmingham, UK. E-mail: jxy223@cs.bham.ac.uk

Guilin Wang is with Huawei International Pte Ltd, Singapore. Email:Wang.Guilin@huawei.com

Yi Mu is with the Centre for Computer and Information Security Research, School of Computer Science and Software Engineering, University of Wollongong, Wollongong, NSW 2522, Australia. E-mail:ymu@uow.edu.au

Wei Gao is with Department of Mathematics and Informatics, Ludong University, Yantai 264025, China; and supported partially by National Natural Science Fundation of China (No.61202475). E-mail:sdgaowei@gmail.com authentication systems remain the most common mechanism for internet applications (e.g. email services, conference management systems, and social networks). However, the security of such systems is not always reliable. For example, poor password selections, the password capture Trojans, and the reuse of passwords could break the security. One popular attack is called dictionary attack, which targets to find the correct password by trying a large amount of likely possibilities, such as words in a dictionary or the likely combination of words. This attack is usually efficient since most users prefer to choose human memorisable passwords, e.g. the user's name, address, or mobile number. A good remedy is additionally using hardware authentication tokens (usually smart cards) to authenticate clients. Such a remedy is called two factor authentication, which has become popular and has been used by applications with higher security guarantees, e.g. internet banking services. In 1991, Chang and Wu [5] introduced this idea of using password and smart card to authenticate clients. Afterwards, many two-factor authentication schemes have been proposed. However, the security of two factor authentication could be compromised since the smart card may be stolen and the data stored in the smart card can be duplicated, and the range of possible passwords could be small and users may forget or lose their passwords. Due to such concerns, biometric identification was introduced to authenticate users by using their biometric features.

In 1999, Juels and Watenberg [6] proposed a biometric authentication scheme, called "fuzzy commitment", that improves some aspects of two-factor authentication because biometric characteristics have higher entropy, and they cannot be forgotten and are rarely to be lost [7]. However, one problem is that biometric characteristics are not completely private since one can "steal" biometric characteristics from others; e.g., the fingerprint can be obtained from a mug that the victim has used, and the facial features may be obtained from a user's photograph. A way to alleviate these problems is to combine all these three factors together. This approach is also known as three-factor authentication, which has been greatly adapted by cloud-based applications (e.g. [8]).

\section{A. Related work}

The introduction of password-based authentication by Lamport in 1981 [4] has inspired numerous password based authentication protocols [9], [10], [11], [12], [13]. In 1999, Yang and Shieh [9] proposed two two-factor authentication 
schemes: one is based on timestamp and the other is based on random nonce. Both of them support contact-less password changing, i.e., users do not need to contact/inform a server to change their password. A system satisfying such requirement can save the computation cost on the server side, and save the communication cost on both server and user side. Later, Chan and Cheng [14], and Fan et al. [10] identified impersonation attacks on the Yang-Schieh scheme. To overcome this flaw, Shen, Lin and Hwang [11], and Yang, Wang and Chang [12] suggested improvements on the Yang-Schieh scheme. However, Yoon et al. [13] showed possible attacks on the YWC-scheme [12], and introduced an improvement. In 2006, however, Wang and Bao [15] pointed out that both the SLHscheme [11] and Yoon et al.'s scheme [13] are vulnerable to impersonation attack.

On the other direction, in 2003, Kim et al. [16] proposed two constructions of three-factor authentication schemes by using password, smart card, and fingerprints, without requiring public key directory tables. However, Scott [17] pointed out that a passive eavesdropper, without accessing to any smart card, password, or fingerprint, could impersonate any identity to pass authentication after successfully eavesdropping only once legitimate log-in.

In 2004, Uludag et al. [18] surveyed various types of biometric authentication systems, and recommended to use digital rights management (DRM) systems [19] to address the problem of biometric authentication systems. In their method, the cryptographic key is bound with a biometric template and stored in a database. Thus, the key cannot be revealed without passing biometric authentication. However, the requirement of the biometric database has increased the cost and put users' privacy at risk. To protect users' privacy, in 2006 BhargavSpantze et al. [20], [21] proposed a novel privacy preserving two-phase three-factor authentication scheme, based on zero knowledge proof (ZKP), in which user privacy is preserved by using the Pedersens commitments [22]. However, the scheme is expensive because of modular exponentiation operations, and the requirement that all users' commitments are stored on the server side. In 2009, Fan and Lin [23] constructed an efficiency enhancing and privacy preserving three-factor authentication scheme, but it does not support contact-less password changing. There are also many other research [24], [25] have been done on preserving user privacy in distributed systems.

Recently, Li and Hwang [26] proposed an efficient three factor user authentication scheme, without requiring synchronized clocks. Later, Li et al. [27] pointed out that the LiHuang scheme does not meet proper authentication since it is vulnerable to the man-in-the-middle attack. To address this shortcoming, they provided a further improvement. In 2011, however, Das et al. [28] found that Li et al.'s improved scheme neither provided strong authentication nor supported contactless password changing. They then proposed an improvement on Li et al.'s scheme. However, the improved scheme is still insecure as an adversary who obtained a victim's smart card can launch off-line password guessing attack.

To tackle the problem caused by insecure proposals and improvements, Huang et al. [29] proposed a generic framework to upgrade two factor authentication schemes to three-factor authentication schemes, while preserving security and privacy. The basic idea is to use a fuzzy extractor to generate the biometric key from the biometric characteristics, and run twice the underlying two-factor authentication scheme. The first run is the normal underlying two-factor scheme using passwords and smart cards. In the second run of the underlying scheme, the password is replaced with the generated biometric key. This framework does not require any change on the underlying two-factor authentication protocol, and in the derived scheme users do not need to hand their biometric characteristics over to the server, so that servers do not need to store any data related to user's biometric characteristics. Thus, user privacy is preserved and the cost on the server side is reduced.

\section{B. Motivation}

Huang et al. [29] offer a good framework to produce threefactor authentication schemes from existing two factor authentication schemes. This framework eases the design of three factor authentication systems, provides higher security guarantee, and preserves user privacy. To generate biometric keys from the biometric characteristics, Huang et al.'s framework employs the "fuzzy extractor" [30]. Fuzzy extractor generates a pair of strings $(P, R)$ from user biometric characteristics, where $P$ is the auxiliary string and $R$ should be kept secret as private key. The private $R$ can be recovered if a user can provide the corresponding auxiliary string $P$ and a close enough biometric characteristics. The error tolerance in the scheme depends on three error correcting techniques, namely Hamming distance, set difference, and edit distance. The fuzzy extractor provides a good insight into biometric identification by extracting a unique and random 'private' key directly from the user's biometric features. However, the fuzzy extractor has not been widely implemented since the distance measures in it are less accepted than the Euclidean distance measurement in biometric applications [31].

Moreover, we observe that the efficiency of Huang et al.'s framework can be improved from running underlying scheme twice to running it once - which saves almost half of the cost in total. Moreover, the study on the concrete three-factor authentication scheme with formally security analysis, which is recognised as an open problem and a challenging issue [32], are missing in their work.

\section{Contributions}

The main contributions of this paper are the improved generic framework for three-factor authentication and a provably secure instantiation. The merits of this paper are as follows.

First, the proposed generic framework enhances efficiency by combining the user's password and the user's biometric key together and using the hash value of this combination as the user's secret key. Consequently, the resulted three-factor scheme only needs to run the underlying two-factor scheme one time. This saves almost half of the communication cost and computation cost for each login among potential billions of users. 
Second, the proposed generic framework is more practical. We employ the improved finger print-based "fuzzy vault" [33] to identify the user's biometric features. Literature shows that the fuzzy extractor has not been implemented yet, while researchers implemented and improved the fuzzy vault scheme in recent years [34], [35], [33], [36], [31]. Moreover, the fuzzy vault has been widely accepted because the Euclidean distance measurement which is used in the fuzzy vault are widely accepted by majority of biometric applications [31]. Therefore, the improved framework selects the fuzzy vault to employ the third factor, biometric features.

Last, a provably secure instantiation is presented. In particular, this paper discusses the practicability analysis of the concrete scheme, compares our concrete scheme with other existing three-factor schemes, provides privacy discussion, and shows formal security proof on the concrete scheme.

\section{Organization}

The rest of this paper is organised as follows. Section II reviews and discusses two well-known biometric identification mechanisms. Section III reviews Huang et al.'s framework and provides an improved generic framework for three-factor authentication. The instantiation with analysis and comparison are given in Section IV. In section V, formal security proof and privacy discussion for this instantiation are provided.

\section{BIOMETRIC IDENTIFICATION MECHANISMS}

In 1999, Juels and Wattenberg [6] proposed "fuzzy commitment", the first biometric identification scheme, which deploys Hamming distance to tolerate errors. Later, Juels and Sudan [37] introduced a provably secure biometric identification scheme, called fuzzy vault, in which a user can generate a long-bit secret key, and encrypt it by using his/her extracted biometric template. The long-bit secret key can be recovered by providing the encrypted data and the corresponding authentic biometric characteristics. In 2003, Clancy et al. [34] proposed a secure smart card based fingerprint authentication scheme by using Juels and Sudan's fuzzy vault. Later, in 2007, Nandakumar et al. [35] proposed a fully automatic implementation by employing the fuzzy vault and using helper data to align unidentified fingerprints accurately. Their scheme used both location $(x, y)$ and orientation attribute $\theta$ of a minutia point to record the biometric data, where $(x, y)$ is the row and column indicators in the image as the location, and $\theta$ is the orientation on the $\mathrm{X}$-axis. The helper data is high curvature points extracted from the fingerprint orientation field, thus it neither affects the security nor leaks any information about the biometric template. One year later, Nagar, Nandakumar and Jain [33] improved the security and matching accuracy of Nandakumar et al's fingerprint-based fuzzy vault scheme by employing additional minutiae descriptors [38], which capture local ridge orientation and ridge frequency information in the neighbourhood of a minutia. The results in [33] show that the improved scheme reduces the false acceptance rate (FAR) and significantly increases the vault security.

On the other direction, in 2004, Dodis et al. [30] proposed fuzzy extractor, which has two procedures: a generation procedure and a reproduction procedure. After a user scanned his biometric features and obtained the biometric template $w$, the generation procedure extracts a random $R$ and a corresponding auxiliary $P$ from $w$. In the authentication phase, the inputs of reproduction procedure are $P$ and an unidentified biometric template $w^{\prime}$; the output of this reproduction procedure is exactly the same $R$ if and only if the difference between $w$ and $w^{\prime}$ is within an acceptable error tolerance. In 2008, Teoh and Ong [39] proposed a randomised dynamic quantisation transformation (RDQT), which is based on fuzzy commitment, to binarize biometric data, and satisfy both randomness and uniqueness. Meanwhile, Sheng et al. [40] presented a template-free biometric-key generation, which can also generate a key directly from a biometric template.

\section{A. Fuzzy vault}

Fuzzy vault is a cryptographic construction for data protection and user authentication, whose security relies on unexposed biometric characteristics and smart card. The error tolerance in fuzzy vault is achieved by using the Euclidean distance measurement which has been widely accepted by the majority of biometric applications. The operations of the fuzzy vault are described as follows.

First, a user extracts biometric template $X$ by scanning her biometric characteristics (e.g. fingerprint). Then, she encodes a pre-self-generated secret string $K$ into a self-selected polynomial $\mathrm{Pol}$, and evaluates the polynomial on all elements in $X$. She also needs to choose a large number of random points which do not lie on $\mathrm{Pol}$ as the noise. The final vault $V$ is the collection of the points which lie on $\mathrm{Pol}$ and the noise points which do not lie on Pol.

She can recover the secret string $K$ from vault $V$ by providing a biometric template $X^{\prime}$ such that the difference between $X$ and $X^{\prime}$ satisfies $\left|X-X^{\prime}\right|<\epsilon$, where $X-X^{\prime}=$ $\left\{x \mid x \in X, x \notin X^{\prime}\right\}$, and $\epsilon$ is an integer which is the fuzziness parameter. This is because that the polynomial $\mathrm{Pol}$ can be reconstructed if a sufficient number of points on $\mathrm{Pol}$ can be identified. Thus, $K$ can be successfully recovered from $P o l$. The detail operation is defined as follows:

Lock:

1) $\underset{K, \text { Pol }}{\stackrel{X}{\rightarrow}} \operatorname{Gen}(\cdot) \rightarrow L$

Taking input a user's biometric template $X$, secret $K$ and polynomial $\operatorname{Pol}, \operatorname{Gen}(\cdot)$ outputs a set $L$ of points which lie on the Pol.

2) $\stackrel{C P}{\longrightarrow} \operatorname{Enc}(\cdot) \rightarrow V$

Taking input $L$ and a set $C P$ of "chaff points" (i.e. random noise points) which do not lie on $\operatorname{Pol}, \operatorname{Enc}(\cdot)$ outputs a vault $V$ such that $V=C P \cup L . C P$ is generated on the user side, and if we denote $r$ the number of points in $L$, and $s$ the number of points in $C P$, then we require $s>>$.

\section{Unlock:}

1) $\stackrel{X^{\prime}}{\rightarrow} \operatorname{Dec}(\cdot) \rightarrow P o l$

Taking input $V$ and biometric template $X^{\prime}, \operatorname{Dec}(\cdot)$ outputs $\mathrm{Pol}$ if and only if $\left|X-X^{\prime}\right|<\epsilon$, where $X-X^{\prime}=\left\{x \mid x \in X, x \notin X^{\prime}\right\}$ and $\epsilon$ is the fuzziness parameter. 
2) $\stackrel{P o l}{\longrightarrow} \operatorname{Rec}(\cdot) \rightarrow K$

Taking input $\mathrm{Pol}, \operatorname{Rec}(\cdot)$ outputs the secret key $K$.

Remark 1: The security of the fuzzy vault is based on the difficulty of distinguishing genuine points from chaff points in vault $V$, and the difficulty to reconstruct the polynomial $\mathrm{Pol}$ in vault $V$. So, the security guarantee is in proportion to the number of added chaff points.

\section{A GENERIC THREE-FACTOR AUTHENTICATION FRAMEWORK}

\section{A. Review of Huang et al.'s framework}

Huang et al.'s framework employs the fuzzy extractor to generate a uniquely long-bit random string as the biometric key for users. By running the underlying two-factor scheme twice, a three-factor scheme is constructed. In particular, the first run uses password and smart card as normal two factor authentication system. In the second run, framework replaces the password by a biometric key and runs the underlying protocol again, thus a three-factor authentication is obtained. Huang et al.'s framework consists of three phases:

\section{Registration:}

The processes of registration includes the following steps:

1) User $U_{i}$ chooses initial password $P W_{1}$ and extracts biometric template $X$ by scanning her biometric features;

2) $U_{i}$ generates a pair $(R, P)$ by providing $X$ to the fuzzy extractor;

3) Let the second password $P W_{2}$ be $h(R)$, where $h(\cdot)$ is a cryptographic hash function;

4) $U_{i}\left[P W_{1}\right] \stackrel{2-\text { Factor-Reg }}{\Longleftrightarrow} S\left[S K_{1}\right] \rightarrow$ Data $_{1}$;

$U_{i}$ runs the underlying two-factor registration protocol (2-Factor-Reg) with initial password $P W_{1}$, and server $S$ uses secret key $S K_{1}$ to generate Data $_{1}$;

5) $U_{i}\left[P W_{2}\right] \stackrel{2-\text { Factor-Reg }}{\Longleftrightarrow} S\left[S K_{2}\right] \rightarrow$ Data $_{2}$.

$U_{i}$ runs the registration protocol again with $P W_{2}$, and $S$ issues $D a t a_{2}$ by using another secret $S K_{2}$;

6) $U_{i}$ obtains a smart card $S C$ which stores $D_{a t a}, D_{1}$ Data $_{2}$, and $\operatorname{Data}_{3}=(P, h(\cdot), \operatorname{Rep}(\cdot))$, where $h(\cdot)$ and $\operatorname{Rep}(\cdot)$ are the corresponding hash function and the reproduction procedure, respectively.

The scheme supposes that $P W_{1}, P W_{2}$ will be deleted immediately from the server side upon completion of the corresponding registration steps. This means that in the registration phase, the server is fully trusted.

\section{Authentication:}

User $U_{i}^{\prime}$ first inserts $S C$ into a card reader, enters her password, and scans her biometric features. We use $X^{\prime}$ to denote the extracted biometric template. The authentication phase is as follows.

1) The smart card recovers $R^{\prime}$ through $\operatorname{Rep}(\cdot)$, and calculates $P W_{2}^{\prime}=h\left(R^{\prime}\right) . R^{\prime}=R$ if and only if $\left|X-X^{\prime}\right|<\epsilon$ for some fuzziness parameter $\epsilon$;

2) $U_{i}^{\prime}\left[P W_{1}^{\prime}\right.$, Data $\left._{1}\right] \stackrel{2-\text { Factor }- \text { Auth }}{\Longleftrightarrow} S\left[S K_{1}\right]$; $U_{i}^{\prime}$ with $\left(P W_{1}^{\prime}, D a t a_{1}\right)$ runs the authentication phase (2Factor-Auth) of the underlying two-factor authentication protocol with server $S$;
3) $U_{i}^{\prime}\left[P W_{2}^{\prime}, D_{a t a}\right] \stackrel{2-\text { Factor-Auth }}{\rightleftharpoons} S\left[S K_{2}\right]$; $U_{i}^{\prime}$ with $P W_{2}^{\prime}, D_{a t a_{2}}$ runs the 2-Factor-Auth with $S$.

The user successfully passes user authentication if and only if both step 2 and step 3 succeeded.

\section{Password Changing:}

The password can be changed by running password changing protocol (2-Factor-Password-Changing) in the underlying twofactor scheme after successfully logging and updating the $S C$ accordingly. The biometrics can be changed by running step 2 and step 3 in the registration phase, then the user and server execute 2-Factor-Password-Changing and update the corresponding data in $S C$.

\section{B. Improved framework}

We assume that the server in the registration phase is trusted. The details are specified as follows:

Three-Factor-Registration: The processes of registration include the following steps:

1) User $U_{i}$ chooses an initial password $P W_{1}$, a long-bit secret key $P W_{2}$.

2) The fuzzy vault device extracts biometric template $X$ by scanning her biometric features.

3) Taking $X, P W_{2}$, and polynomial $P o l$ as inputs, $\operatorname{Gen}(\cdot)$ outputs a set $L$, and by taking the set $C P$ of noise chaff points and $L$, the Ence $(\cdot)$ outputs the encrypted data $V$.

4) $U_{i}[P W] \stackrel{2-\text { factor-Reg }}{\Longleftrightarrow} S[S K] \rightarrow$ Data $_{1}$, where $P W=h\left(P W_{1} \| P W_{2}\right)$ and $\|$ is concatenation operation. The user with $P W$ and the server with $S K$ run the registration phase of the underlying protocol.

5) Server stores Data $a_{1}$ and $\operatorname{Data}_{2}=$ $(V, \operatorname{Rec}(\cdot), \operatorname{Dec}(\cdot), h(\cdot)) \quad$ in $\quad$ smart $\quad$ card $\quad S C$, and gives it to $U_{i}$.

Three-Factor-Authentication:

To access services, user $U_{i}^{\prime}$ inserts $S C$ to a card reader, which can extracts the data from the $S C$. Then, $U_{i}^{\prime}$ inputs $P W_{1}^{\prime}$ and scans her biometric features, the extracted biometric template is $X^{\prime}$. The details are as follows:

1) The card reader extracts $X^{\prime}$ from $U_{i}^{\prime}$ 's biometric features, and reproduces $P W_{2}^{\prime}$ such that $P W_{2}^{\prime}=P W_{2}$ if and only if $\left|X-X^{\prime}\right|<\epsilon$;

2) The smart card calculates $P W^{\prime}=h\left(P W_{1}^{\prime} \| P W_{2}^{\prime}\right)$;

3) $U_{i}^{\prime}\left[P W^{\prime}, D_{a t a}\right] \stackrel{2-\text { factor-Auth }}{\Longleftrightarrow} S[S K]$;

The user can successfully pass authentication if and only if this step is success.

\section{Three-Factor-Password-Changing:}

The $P W_{1}$ can be changed by following steps.

1) After passing authentication, $U_{i}^{\prime}$ sends the password changing request, inputs new password $P W_{1}^{\prime \prime}$, and scans the biometric template.

2) The 'fuzzy vault' device will recover the $P W_{2}$ by using the 'fuzzy vault' decoding scheme.

3) The smart card calculates $P W^{\prime \prime}=h\left(P W_{1}^{\prime \prime}|| P W_{2}\right)$.

4) $P W^{\prime \prime}$ is taken as the password and runs the password changing phase of the underlying protocol.

Biometric key $P W_{2}$ can be changed in a similar way. For this purpose, $U_{i}^{\prime}$ chooses a new biometric key as $P W_{2}^{\prime \prime}$, 
then encrypts it via the fuzzy vault device, outputs $V^{\prime}$ which replaces current $V$ in $S C$. The $S C$ calculates $P W^{\prime \prime}=$ $h\left(P W_{1} \| P W_{2}^{\prime \prime}\right)$, then takes $P W^{\prime \prime}$ as the password and runs the password changing phase of the underlying protocol.

\section{INSTANTIATION}

Our instantiation will use the Yang et al.'s two-factor authentication scheme [41], which is provably secure, as the underlying scheme.

Let $G$ be a group of prime order $q$ and $g$ a generator, $H:\{0,1\}^{*} \rightarrow\{0,1\}^{k}$ denote a collision resistant hash function, $H^{\prime}:\{0,1\}^{*} \rightarrow\{0,1\}^{k}$ a hash function which preserves the entropy of its input (e.g. add paddings after the input); $P R F_{K}:\{0,1\}^{k} \rightarrow\{0,1\}^{k}$ a pseudo-random function keyed by $K$. In addition, we assume that a server $S$ has a long term secret $x$ such that $x \in\{0,1\}^{k}$, and encryption and signature key pairs $(P K, S K)$ and $\left(P K^{\prime}, S K^{\prime}\right)$, respectively.

Let $E_{P K}(M)$ denotes the asymmetric key encryption on message $M$ under public key $P K ; \operatorname{Sig}_{S K^{\prime}}(M)$ a signature on $M$ issued by using signing key $S K^{\prime}$.

\section{A. Review of Yang et al.'s scheme}

In the registration phase, a user $U_{i}$ chooses a unique identity $I D_{i}$ and sends it to the server $S$. After receiving the request, $S$ issues a credential $C_{i}=P R F_{x}\left(H\left(I D_{i}\right)\right)$, and hides it by calculating $B=C_{i} \oplus H^{\prime}\left(P W_{0}\right)$, where $P W_{0}$ is the initial password chosen by $S$; then $S$ sends the initial password $P W_{0}$ and a smart card which contains $\left(P K, P K^{\prime}, I D_{i}, B, p, g, q\right)$ to $U_{i}$.

The login phase is presented in the Fig. 1. To log in, $U_{i}$ attaches her smart card to a card reader device, and enters her password $P W^{\prime}$. The smart card calculates $C_{i}^{\prime}=B \oplus H^{\prime}\left(P W^{\prime}\right)$ and sends $\left(I D_{i}, s i d, g^{a}\right)$ to $S$, where sid is the session identifier, and $a$ is a new selected random number. $S$ should send $\left(S I D, I D_{i}\right.$, sid, $g^{b}, \operatorname{Sig}_{S K^{\prime}}\left(S I D, I D_{i}\right.$, sid $\left.\left., g^{a}, g^{b}\right)\right)$ to $U_{i}$, where $S I D$ is the identity of $S$ and the signature is used for server side authentication. If the signature is valid, then $U_{i}$ believes that he is talking to the real server, and sends $\left(I D_{i}\right.$, sid, $\left.C T\right)$ to $S$, where $C T=E_{P K}\left(C_{i}^{\prime}, I D_{i}, S I D\right.$, sid $\left.\left., g^{a}, g^{b}\right)\right) . S$ accepts $U_{i}$ as a genuine user if $C_{i}^{\prime}=P R F_{x}\left(H^{\prime}\left(I D_{i}\right)\right)$. Now both parties believe that they have shared the same session key $g^{a b}$.

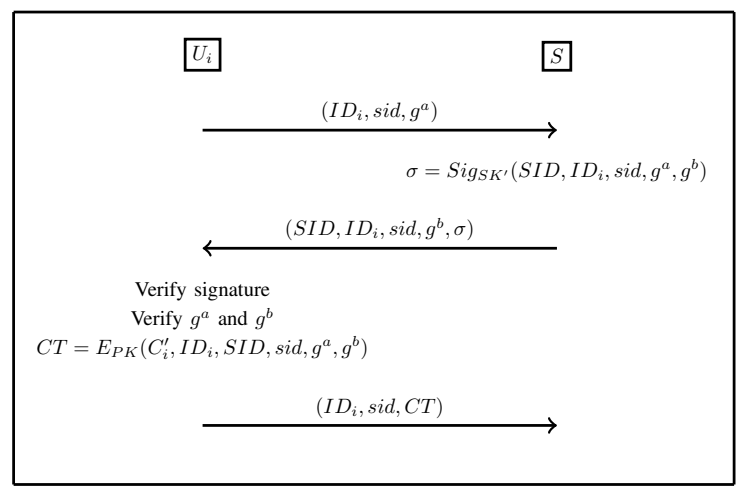

Fig. 1. Login phase of Yang et al.'s scheme
In addition, $U_{i}$ can change her password at anytime after she receiving the smart card and initial password $P W_{0}$ from $S$. To change the password, she picks a new password $P W_{\text {new }}$, and performs $B_{\text {new }}=B \oplus H^{\prime}\left(P W_{0}\right) \oplus H^{\prime}\left(P W_{\text {new }}\right)$, then replaces $B$ with $B_{\text {new }}$.

\section{B. Protocol}

The basic idea of our concrete protocol is that using $P W=$ $H^{\prime}\left(P W_{1} \| P W_{2}\right)$ as the password in Yang et al's scheme, where $P W_{1}$ is the real password, and $P W_{2}$ is the biometric key encrypted through fuzzy vault scheme. A user can pass authentication only if s/he provides the correct password, smart card, and the biometric features which is close enough with the one used in the registration phase.

\section{Registration}

In the registration phase, a user $U_{i}$ performs exactly the same as in Yang et al.'s scheme. However, after $U_{i}$ receiving the smart card and the initial password $P W_{0}$, she needs to additionally selects a new password $P W_{1}$, a polynomial $P o l$ and a biometric key $P W_{2}$. In addition, she extracts her biometric template $X$, encrypts $P W_{2}$ through fuzzy vault device which outputs a vault $V$. Then $U_{i}$ writes $V$ into the smart card, and calculates $P W=H^{\prime}\left(P W_{1} \| P W_{2}\right)$, and updates $B$ by computing $B=C_{i} \oplus H^{\prime}\left(P W_{0}\right) \oplus P W$. The 'fuzzy vault' procedures are reviewed in the Section II-A, thus we omit the detail here.

\section{Login-and-Authentication Phase}

User $U_{i}^{\prime}$ attaches her smart card to a card reader device, inputs password $P W_{1}^{\prime}$ and scans her biometric features. The fuzzy vault device extracts the biometric template $X^{\prime}$, then the fuzzy vault device calculates $P o l^{\prime}=\operatorname{Dec}\left(X^{\prime}, V\right)$, and $P W_{2}^{\prime}=$ $\operatorname{Rec}\left(P \operatorname{Pl}^{\prime}\right)$. The smart card $S C$ calculates $C_{i}^{\prime}=B \oplus P W^{\prime}$, where $P W^{\prime}=H^{\prime}\left(P W_{1}^{\prime} \| P W_{2}^{\prime}\right)$. Then, the protocol runs the login phase as the same as Yang et al's scheme by using $P W^{\prime}$.

\section{Password-Changing}

To change an old password $P W_{1}, U_{i}$ performs the following steps.

1) Chooses a new password $P W_{1}^{\prime}$.

2) Calculates $P W_{\text {new }}=H^{\prime}\left(P W_{1}^{\prime} \| P W_{2}\right)$ and computes $B_{\text {new }}=B \oplus P W \oplus P W_{\text {new }}$, where $P W=$ $H^{\prime}\left(P W_{1} \| P W_{2}\right)$.

3) Replace $B$ with $B_{\text {new }}$ in the smart card.

The biometric key $P W_{2}$ and the biometric features can be changed in a similar way, in which case, the vault $V$ in the smart card should also be updated.

\section{Analysis of implementation}

To analyze the derived three-factor authentication scheme, we take the fingerprint based fuzzy vault scheme [35] proposed by Nandakumar, Jain, and Pankanti in 2007, though any secure biometrics authentication protocol can be used. In their fuzzy vault scheme, each element $v_{i} \in V(i \in\{1,2, \ldots, r+s\})$ is represented as three-tuple such that $v_{i}=(x, y, \theta)$, where $r$ is the number of points in $L$ (w.r.t. the points in $V$ which lie on $P$ ) and $s$ denotes the number of noise points in $V$ which do not lie on $P,(x, y)$ is the row and column coordinates in the 
image showing the location, $\theta$ is the orientation which respect to the $\mathrm{X}$-axis.

In addition, we take $s \approx 10 r$ to satisfy the requirement that $s>r$. Moreover, 8-degree polynomial is used to encrypt 128-bit secrets, and the lengths of $x, y, \theta$ (quantized and represented in bit strings) are $6,5,5$, respectively. As the parameter showed in [35], there are around 30 points which lie on the selected polynomial in a $640 \times 480$ at 500 dpi resolution fingerprint image, so we could conclude $r=30$ and $s=300$. Thus, $V$ contains 330 points which requires 660 Bytes space. Furthermore, the length of help data used in this fuzzy vault scheme is depended on the points of maximum curvature in the flow curves, and it can be ignored. Thus, only less than $1 \mathrm{~KB}$ additional data are required if compared with the underlying two factor authentication scheme.

The genuine acceptance rate (GAR) and false acceptance rate (FAR) are influenced by the degree of polynomial. In the above setting, the FAR falls in $0.01 \%-0.04 \%$ and GAR is grater than $90 \%$. In fact, GAR is acceptable even if $G A R=$ $50 \%$, as this means that genuine users can pass authentication by scanning their fingerprint about twice.

We compare our instantiation with other schemes into two tables, namely Table I and Table II. The focus of the first table is on the efficiency, and the second table is mainly focusing on the security and privacy. In Table I, Reg-Cost and Auth-Cost present the computational cost in the registration phase and authentication phase, respectively; the number indicates that how many times the corresponding operation is required by the protocol, e.g. 2 EXP means that exponentiation computation is required twice.

These two tables show that Li-Hwang scheme [26], Li et al.'s scheme [27], Das's scheme [28], and Kim-Lee-Yoo scheme [16] support contactless password changing, and the first three schemes only have very small computation cost. However, all of them have security flaws. In contrast, both Bhargav-Spantze et al.'s scheme [21] and Fan-Lin scheme [23] are secure under the three-factor adversary model, but they do not support contactless password changing and BhargavSpantze et al.'s scheme does not support session key establishment, so perfect forward secret cannot been guaranteed. While the derived protocol protects user privacy, offers contactless password changing, and supports session key establishment, with acceptable computation cost.

\section{SECURITY AND PRIVACY ANALYSIS}

The hypothesis of the security of our proposed generic framework is that (A) the underlying two-factor authentication protocol is secure when any one factor is compromised, and (B) the fuzzy vault system is secure when the biometric template is kept secret. In a system derived by using our framework, the authentication process is actually the same as the underlying two-factor authentication protocol. However, the difference is that, the "password" $P W=h\left(P W_{1} \| P W_{2}\right)$ is the output of a hash function, where the input data are the human memorisable password $P W_{1}$, and the secret bitstring $P W_{2}$ which is protected by using the fuzzy vault system.

\footnotetext{
${ }^{1}$ This guarantees that the server does not store users' password and biodata.
}

Considering three different cases: the $P W_{1}$ and the biometric template are exposed to the attacker, the $P W_{1}$ and the smart card are exposed to the attacker, and the biometric template and the smart card are exposed to the attacker.

To make the analysis easier to be understood, we assume a very strong attacker, who can recover $P W_{2}$ if the biometric template is compromised, though actually the attacker also needs the information stored in the smart card. However, this assumption will not affect our security since if the system is secure against a very strong attacker, then the system is also secure against a weak attacker.

Loosely speaking, if the $P W_{1}$ and biometric template (so the $P W_{2}$ ) are compromised, then it is the similar case as that in the underlying two-factor authentication protocol, the password is corrupted while the smart card remains secure (since $P W$ can be computed in this case). So the derived system will remain secure. Otherwise, we can build a probabilistic polynomial time (PPT) Turing machine to break the security of the underlying two-factor authentication protocol, which contradicts to the hypothesis (A).

If the case that $P W_{1}$ and the smart card are compromised, we have that $P W_{2}$ is secure thanks to the hypothesis (B). In addition, by hypothesis (A), we have that the system is secure if $P W$ remains secure. So, the only way the attacker can pass the authentication is to discover the value of $P W$. If there is a way to discover the value of $P W$ with overwhelming probability, then we can either construct a PPT Turing machine that is able to discover the value of the password in the underlying two-factor authentication protocol, which is a contradiction of hypothesis (A); or we can find the hash collision which contradicts to the assumption of a secure hash function. The case that the biometric template and smart card are composed is similar to this case. Now, we present the formal security analysis of the instantiation given in Section IV.

Considering two communicating parties $A$ and $B$, a mutual authentication protocol is secure if and only if participant $A$ accepting participant $B$ implies $B$ accepting $A$. The generic security model of mutual authentication have been well studied [42], [43], [44]; however, more strict security model is desired for the three-factor authentication systems due to the more intricate authentication conditions. Currently, the formal security analysis of multiple factor authentication scheme remains as a challenging issue [32], although there are some existing works [45], [23], [46].

This section proposes a security model for three-factor authenticated key exchange schemes by extending and adopting the existing generic model [42]. Based on the proposed model, we prove the security of the derived scheme.

\section{A. Security model}

We place probabilistic polynomial time (PPT) adversary $A$ between user $U_{i}$ in user set $U$ and sever $S_{j}$ in server set $S$. Let $\prod_{U, S}^{s i d}$ be the user oracle interacting with the server in session sid; and $\prod_{S, U}^{s i d}$ denotes the server oracle interacting with user in the session sid. It is obvious that if protocol $\prod$ is secure when $A$ knows two out of three factors, then $\prod$ is still secure when only one factor has been leaked to $A$. Therefore, we 
TABLE I

COMPARISON OF SCHEMES (A)

\begin{tabular}{|c|c|c|c|c|c|c|c|c|}
\hline Efficiency & Scheme & $\begin{array}{c}\text { Li-Hwang } \\
\text { scheme [26] }\end{array}$ & $\begin{array}{c}\text { Li et al.'s } \\
\text { scheme [27] }\end{array}$ & $\begin{array}{c}\text { Das's } \\
\text { scheme [28] }\end{array}$ & $\begin{array}{l}\text { Kim-Lee-Yoo } \\
\text { scheme [16] }\end{array}$ & $\begin{array}{l}\text { Bhargav-Spantze } \\
\text { et al.'s scheme [21], [20] }\end{array}$ & $\begin{array}{c}\text { Fan-Lin } \\
\text { scheme [23] }\end{array}$ & $\begin{array}{l}\text { Proposed } \\
\text { scheme }\end{array}$ \\
\hline \multicolumn{2}{|c|}{ No server storage $^{1}$} & $\sqrt{ }$ & $\sqrt{ }$ & $\sqrt{ }$ & $\sqrt{ }$ & X & X & $\sqrt{ }$ \\
\hline \multirow{2}{*}{ Reg-Cost } & Server & Very Low & Very Low & Very Low & 2 EXP & $1 \mathrm{EXP}$ & Low & Low \\
\hline & Client & Very Low & Very Low & Very Low & Very Low & $2 \mathrm{EXP}$ & Low & Very low \\
\hline \multirow{2}{*}{ Auth-Cost } & Server & Very Low & Very Low & Very Low & $2 \mathrm{EXP}$ & 3 EXP & $1 \mathrm{PKD}$ & 1 SigSign, 2 EXP, 1 PKD \\
\hline & Client & Very Low & Very Low & Very Low & 2 EXP & $2 \mathrm{EXP}$ & PKE & 1 SigVer, 2 EXP, 1 PKE \\
\hline
\end{tabular}

Very Low The most expensive operation is hash function.

Low The most expensive operation is symmetric key encryption/decryption.

EXP: Large exponentiation computation is required.

PKE (or PKD): Asymmetric key encryption (or decryption) is required.

SigSign (or SigVery): Digital signature signing (or verification) is required.

TABLE II

COMPARISON OF SCHEMES (B)

\begin{tabular}{|c|c|c|c|c|}
\hline Scheme & $\begin{array}{c}\text { Contactless } \\
\text { password } \\
\text { changing }\end{array}$ & $\begin{array}{c}\text { Biometrics } \\
\text { privacy }\end{array}$ & $\begin{array}{c}\text { Session key } \\
\text { establishment } \\
\text { supportance }\end{array}$ & Security \\
\cline { 1 - 4 } Li-Hwang scheme [26] & $\sqrt{ }$ & $\times$ & $\times$ & Vulnerable to man-in-the-middle attack \\
\hline Li et al.'s scheme [27] & $\sqrt{ }$ & $\times$ & $\sqrt{ }$ & Fail to provide strong authentication \\
\hline Das's scheme [28] & $\sqrt{ }$ & $\times$ & $\sqrt{ }$ & Vulnerable to off-line password guessing attack \\
\hline Kim-Lee-Yoo scheme [16] & $\sqrt{ }$ & $\sqrt{ }$ & $\times$ & Vulnerable to impersonation attack \\
\hline $\begin{array}{c}\text { Bhargav-Spantze } \\
\text { et al.'s scheme [21], [20] }\end{array}$ & $\times$ & $\sqrt{ }$ & $\times$ & Secure under three-factor requirements \\
\hline Fan-Lin scheme [23] & $\times$ & $\sqrt{ }$ & $\sqrt{ }$ & Secure under three-factor requirements \\
\hline Proposed scheme & $\sqrt{ }$ & $\sqrt{ }$ & $\sqrt{ }$ & Secure under three-factor requirements \\
\hline
\end{tabular}

only consider the case of two corrupted factors. $A$ can make following oracle queries.

1) Register $\left(\prod, S_{j}\right)$ : Upon receiving this query from $A$, the server oracle acts as $S_{j}$ to run the registration phase with $A$, and issues identity $I D_{i}$ and sends smart card $S C$ to $A$.

2) Execute $\left(U_{i}, S_{j}\right.$, sid $)$ : This oracle query models all passive attackers who can eavesdrop on all messages transmitted between $U$ and $S$ in session sid in $\Pi$. Upon receiving this query, $\prod_{U, S}^{s i d}$ and $\prod_{S, U}^{s i d}$ will execute protocol as $U_{i}$ and $S_{j}$ in $\prod$, respectively. The messages exchanged between them will be recorded and sent to $A$.

3) $\operatorname{Send}\left(U_{i}, S_{j}\right.$, sid $\left., M_{m}, m\right)$ : This query sends message $M_{m}$ with sequence of message flow $m$ to server oracle $\prod_{S, U}^{s i d}$ which simulates $S_{j}$, and then, the oracle will compute a response honestly in $\Pi$, and send the response to $A$.

4) $\operatorname{Send}\left(S_{j}, U_{i}\right.$, sid $\left., M_{m^{\prime}}, m^{\prime}\right)$ : This query sends message $M_{m^{\prime}}$ with a sequence of message flow $m^{\prime}$ to user oracle $\prod_{U, S}^{s i d}$ which simulates $U_{i}$, and then, the user oracle will compute a response honestly in $\prod$, and send the response to $A$. Upon receiving the query with $m^{\prime}=\lambda$, where $\lambda$ is an empty set, from $A$, the user oracle will start a new session and send a service request message to $A$.

5) Reveal $\left(\prod, U_{i}, S_{j}\right.$, sid): This query models the leakage of a session key in session sid between user $U_{i}$ and server $S_{j}$. This query can only be made when a session key has been shared between the server and the user in session sid. Upon receiving this query, the user oracle will send the shared session key to $A$.

6) There are three corruption queries:

a) $\operatorname{Corrupt}\left(U_{i}, p w, S C\right)$. Upon receiving this query, user oracle will output the user $U_{i}$ 's password $p w$ and the data stored in the smart card $S C$;

b) $\operatorname{Corrupt}\left(U_{i}, p w, B i o\right)$. Upon receiving this query, user oracle will output the user $U_{i}$ 's password $p w$ and the biometric template $B i o$;

c) $\operatorname{Corrupt}\left(U_{i}, S C, B i o\right)$. Upon receiving this query, user oracle will output the user $U_{i}$ 's biometric template Bio and the data stored in the smart card $S C$;

Note that $A$ can only make one corruption query on the same target.

7) $\operatorname{Test}\left(U_{i}, S_{j}\right.$, sid): This query can be made by $A$ only after a session key has been shared between $U_{i}$ and $S_{j}$ in a fresh session sid. If so, then a coin $b$ is tossed, if it lands $b=0$, then this oracle outputs the session key. Otherwise, a fixed-length random string is returned. 
$A$ needs to output $b^{\prime}=0$ or $b^{\prime}=1$ as the result of distinguishing the session key from the random string. $A$ can only ask this query once.

The definitions of matching conversations, secure mutual authentication and secure key exchange [42] are reviewed as follows.

Definition 1: (Matching Conversations): Considering fix number of moves $R=2 \rho-1$ and $R$-move protocol $\prod$. Run $\prod$ in the presence of adversary $A$ and consider two oracles $\prod_{U, S}^{s i d}$ and $\prod_{S, U}^{\text {sid }}$ that engage in conversations $K$ and $K^{\prime}$, respectively. $(\tau, \alpha, \beta)$ denotes that $A$ obtains response $\beta$ by sending $\alpha$ to an oracle at time $\tau . \alpha_{1}=\lambda$ indicates the start point of a new session in protocol $\prod$. "*” denotes the final decision of $R$-move protocol $\prod$.

1) We say that $K^{\prime}$ is a matching conversation to $K$ if there exist $\tau_{0}<\tau_{1}<\ldots<\tau_{R}$ and $\alpha_{1}, \beta_{1}, \ldots, \alpha_{\rho}, \beta_{\rho}$ such that $K$ is prefixed by $\left(\tau_{0}, \lambda, \alpha_{1}\right),\left(\tau_{2}, \beta_{1}, \alpha_{2}\right)$, $\ldots,\left(\tau_{2 \rho-4}, \beta_{\rho-2}, \alpha_{\rho-1}\right),\left(\tau_{2 \rho-2}, \beta_{\rho-1}, \alpha_{\rho}\right)$ and $K^{\prime}$ is $\left(\tau_{1}, \alpha_{1}, \beta_{1}\right),\left(\tau_{3}, \alpha_{2}, \beta_{2}\right), \ldots,\left(\tau_{2 \rho-3}, \alpha_{\rho-1}, \beta_{\rho-1}\right)$.

2) We say that $K$ is a matching conversation to $K^{\prime}$ if there exist $\tau_{0}<\tau_{1}<\ldots<\tau_{R}$ and $\alpha_{1}, \beta_{1}, \ldots, \alpha_{\rho}, \beta_{\rho}$ such that $K^{\prime}$ is prefixed by $\left(\tau_{1}, \alpha_{1}, \beta_{1}\right),\left(\tau_{3}, \alpha_{2}, \beta_{2}\right), \ldots,\left(\tau_{2 \rho-3}, \alpha_{\rho-1}, \beta_{\rho-1}\right)$, $\left(\tau_{2 \rho-1}, \alpha_{\rho}, *\right)$ and $K$ is $\left(\tau_{0}, \lambda, \alpha_{1}\right),\left(\tau_{2}, \beta_{1}, \alpha_{2}\right), \ldots$, $\left(\tau_{2 \rho-4}, \beta_{\rho-2}, \alpha_{\rho-1}\right),\left(\tau_{2 \rho-2}, \beta_{\rho-1}, \alpha_{\rho}\right)$.

Let $\prod_{U_{i}, S_{j}}^{\text {sid }}$ (resp. $\prod_{S_{j}, U_{i}}^{\text {sid }}$ ) be the oracle which acts as user $U_{i}$ (resp. server $S_{j}$ ) communicating with server $S_{j}$ (resp. user $U_{i}$ ). Let $N_{o}-$ Matching $^{A, U_{i}}(k)$ (resp. NoMatching $^{A, S_{j}}(k)$ ) be the event that there exist $U_{i}, S_{j}$ and sid such that $\prod_{U_{i}, S_{j}}^{s i d}$ (resp. $\prod_{S_{j}, U_{i}}^{s i d}$ ) has accepted $A$ as $\prod_{S_{j}, U_{i}}^{s i d}$ (resp. $\prod_{U_{i}, S_{j}}^{s i d}$ ), while $\prod_{S_{j}, U_{i}}^{s i d}$ (resp. $\prod_{U_{i}, S_{j}}^{s i d}$ ) has not engaged in a matching conversation. In other words, it is the event that user $U_{i}$ (resp. server $S_{j}$ ) believes that server $S_{j}$ (resp. user $U_{i}$ ) is communicating with him, but in fact, it is the adversary $A$ who has impersonated server $S_{j}$ (resp. user $U_{i}$ ).

Remark 2: The above definition is defined for the case of $R=2 \rho-1$. The case of $R=2 \rho$ is similar and we omit it here.

Definition 2: (Secure Three-Factor Mutual Authentication $(S T M A))$ We say that $\prod$ is a secure mutual authentication protocol if the following properties are satisfied in presence of PPT adversary $A$ defined in the adversary model.

1) If oracle $\prod_{U_{i}, S_{j}}^{\text {sid }}$ and $\prod_{S_{j}, U_{i}}^{i}$ have matched conversations, then they accept each other.

2) $\prod_{U_{i}, S_{j}}^{\text {sid }}$ accepted implies a matching conversation: the probability of $N_{o}-\operatorname{Matching}^{A, U_{i}}(k)$ is negligible, where $S_{j}$ should not be registered by $A$. (Secure server authentication)

3) $\prod_{S_{j}, U_{i}}^{s i d}$ accepted implies a matching conversation: the probability of $N_{o}-$ Matching ${ }^{A, S_{j}}(k)$ is negligible, where $U_{i}$ should not be registered by $A$. (Secure user authentication)

Definition 3: (Secure Three-Factor Authenticated Key Exchange $(S T A K E))$ A Protocol $\prod$ is called $S T A K E$ if the following properties hold in presence of PPT adversary $A$ defined in the adversary model:
- $\Pi$ is an $S T M A$ protocol;

- if the session is fresh in protocol $\prod$, and both $\prod_{U_{i}, S_{j}}^{s i d}$ and $\prod_{S_{j}, U_{i}}^{i}$ complete matching conversations, then they have shared the same session key;

- the advantage $A d v^{A}(k)$ is negligible.

Note that:

- A session is called fresh if both $\prod_{U_{i}, S_{j}}^{s i d}$ and $\prod_{S_{j}, U_{i}}^{s i d}$ accepted each other and no session key reveal query has been made to $\prod_{U_{i}, S_{j}}^{s i d}$ or $\prod_{S_{j}, U_{i}}^{\text {sid }}$.

- $A d v^{A}(k)=\left|\operatorname{Pr}\left[\operatorname{Gguess}^{A}(k)\right] \quad-\frac{1}{2}\right|$, where the $\operatorname{Pr}\left[\operatorname{Gguess}^{A}(k)\right]$ is the probability such that $A$ has won in the $T e s t\left(U_{i}, S_{j}\right.$, sid $)$.

\section{B. Formal security analysis}

To prove the security of our concrete scheme, we shall show that if $A$ can successfully pass user or server authentication with a non-negligible probability, then we can construct a PPT Turing machine $T$ to solve the underlying hard problem under the help of $A$ with a non-negligible probability. The concrete protocol is reviewed as follows:

1) $U_{i} \rightarrow S: \quad M_{1}=\left(I D_{i}\right.$, sid,$\left.g^{a}\right)$

2) $S \rightarrow U_{i}: M_{2}=\left(S I D\right.$, sid, $g^{b}, \operatorname{Sig}_{S K^{\prime}}\left(S I D, I D_{i}\right.$, sid, $\left.\left.g^{a}, g^{b}\right)\right)$

3) $U_{i} \rightarrow S: \quad M_{3}=\left(I D_{i}\right.$, sid, $\left.C T\right)$, where $C T=E_{P K}\left(C_{i}^{\prime}\right.$, $I D_{i}, S I D$, sid $\left., g^{a}, g^{b}\right)$

4) $S$ checks credential $C_{i}^{\prime}$. $U_{i}$ will pass user authentication if and only if $C_{i}^{\prime}=P R F_{x}\left(H\left(I D_{i}\right)\right)$.

Now, the shared session key is $g^{a b}$.

Lemma 1: (Secure User Authentication) In the proposed protocol $\prod$, if the pseudo-random function (PRF) is replaced by an ideal random function, the public key encryption scheme is secure against CCA2 attack, and $\prod_{S_{j}, U_{i}}^{\text {sid }}$ has accepted, then the probability of $N o-$ Matching ${ }^{A, S_{j}}(k)$ is negligible even in presence of PPT adversary $A$ in the adversary model.

Proof: This can be proved by contradiction. If there exists an adversary $A$ who can pass user authentication with nonnegligible probability $\epsilon$, then we can construct a PPT Turing machine $T$ to solve the underlying hard problem without knowing secret key $x$, i.e. winning the game of PRF (GamePRF), with a non-negligible probability by using $A$.

Let's assume that $P R F$ is an ideal random function. The Game-PRF is defined as follows: there are two participants, a challenger and a PRF oracle $\prod_{P R F}$ which has the secret $x$. The challenger has the power to ask $\prod_{P R F}$ for the $P R F_{x}(M)$ of any message $M$ as many times as she wants. The game is that this challenger sends two different plaintexts $P_{0}$ and $P_{1}$ to the $P R F$ oracle, which will output $P R F_{x}\left(P_{b}\right)$ to the challenger, where $P_{0}$ and $P_{1}$ have not been asked by the challenger, and $b$ is either 0 or 1 according to the result of coin tossing. After that, the challenger needs to output $b^{\prime}=0$ or $b^{\prime}=1$ as her guess of value $b$. If $b^{\prime}=b$, then the challenger won the game. Let $\operatorname{Pr}_{a d v}[P R F]=\operatorname{Pr}_{w i n}-\frac{1}{2}$ be the advantage of correct guessing of $b$, where $\operatorname{Pr}_{\text {win }}$ denotes the probability of the event that this challenger won the game.

The basic idea is that to win Game-PRF, $T$ simulates an environment of our concrete protocol to convince adversary 
$A$ that this simulation is the real environment of concrete protocol execution. On the other side, $A$ should only has a negligible probability to know the truth, i.e. this is not a real protocol environment but a simulation. In such a simulation, $T$ communicates with $A$ who has the ability to break our concrete protocol in some way in a session with session ID sid with a non-negligible probability. Then, in order to win Game-PRF, $T$ will make use of $A$ 's ability to make the decision of which input message has been used to generate the output $P R F_{x}\left(P_{b}\right)$ with a non-negligible probability.

The simulation is constructed as follows. In the simulation, $T$ answers all oracle queries made by $A$. To achieve this goal, $T$ needs to setup $(S K, P K)$ for the public key scheme and $\left(S K^{\prime}, P K^{\prime}\right)$ for the signature scheme, while $T$ does not know the value of long term secret key $x$ which is for $\prod_{P R F} \cdot \prod_{U_{i}, S_{j}}^{s i d}$ denotes the user oracle which has password $P W_{1}$, smart-card $S C$, and corresponding biometric template $X$ which can recover biometric key $P W_{2}$ with the $S C \cdot \prod_{S_{j}, U_{i}}^{s i d}$ denotes the server oracle which has $P R F$ oracle $\prod_{P R F}$. In our concrete protocol, $A$ can make the following queries:

- Register $\left(\prod, S_{j}\right)$ : Upon receiving this query from $A$, $T$ runs the registration phase with $A$ with the help of $\prod_{P R F}$.

- Execute $\left(U_{i}, S_{j}\right.$, sid $)$ : In $\prod, \prod_{U_{i}, S_{j}}^{s i d}$ and $\prod_{S_{j}, U_{i}}^{\text {sid }}$ generate and record all messages transmitted between $U_{i}$ and $S_{j}$ in session sid, then send these messages to $A$.

- $\operatorname{Send}\left(U_{i}, S_{j}, \operatorname{sid}, M_{m}, m\right): A$ can send $M_{1}$ to $T$, then $T$ responds to $M_{2}$ by using $S K^{\prime}$ to sign a signature as the protocol specified. Upon receiving $M_{3}$ from $A, T$ sends the result of user authentication according to $M_{1}$ and $M_{3}$ by using $S K$ to decrypt the ciphertext and asking $\prod_{P R F}$ in order to verify the credential.

- $\operatorname{Send}\left(S_{j}, U_{i}, \operatorname{sid}, M_{m^{\prime}}, m^{\prime}\right)$ : Upon receiving a new session query $\operatorname{Send}\left(S_{j}, U_{i}\right.$, sid, $\left.M_{\lambda}, \lambda\right), T$ asks $\prod_{U_{i}, S_{j}}^{s i d}$ to send first message $M_{1}$ to $A$. After receiving corresponding message $M_{2}, T$ checks the signature by using $P K^{\prime}$. If the signature is valid, $T$ asks $\prod_{P R F}$ and encrypts its output to form message $M_{3}$.

- $\operatorname{Corrupt}\left(U_{i}\right.$, factor $_{a}$, factor $\left._{b}\right)$ : Upon receiving this query, $\prod_{U_{i}, S_{j}}^{s i d}$ will send the corresponding two factors according to $a$ and $b$, where $a, b \in\{p w, S C, B i o\}$ and $a \neq b$.

If $A$ can pass user authentication successfully with a non-negligible probability without asking $\prod_{U_{i}, S_{j}}^{s i d}$, there must exist a matching conversation between $A$ and $T$ who simulates server $S_{j}$ if the following happens. First, $A$ asks $\operatorname{Corrupt}\left(U_{i}\right.$, factor $_{a}$, factor $\left._{b}\right)$ to obtain two factors, then sends the first message to $T$ who then responds with the second message. Finally, $A$ forms the third message to $T$.

Now, we show how $T$ makes use of $A$ to win Game-PRF with non-negligible advantage as follows. We assume that $A$ attacks at least once among $q_{s}$ sessions, while $T$ does not know which session $A$ is going to attack. Now, $T$ chooses a session out of $q_{s}$ sessions randomly. Then, the probability of $A$ passing user authentication in this session is $\frac{1}{q_{s}} \cdot \epsilon$.

To avoid the case that $A$ found that this environment is only a simulation, in the rest $q_{s}-1$ sessions, $T$ redirects the identity $I D_{r}$, which is included in the first message, to oracle $\prod_{P R F}$ which will respond $P R F_{x}\left(I D_{r}\right)$ back to $T$. Then, $T$ records this identity into the compromised table and checks whether $A$ has passed the user authentication by matching $P R F_{x}\left(I D_{r}\right)$ with the credential which is encrypted in the third message. If they are matched, then $T$ responds to $A$ that $T$ accepts $A$ 's login request. Otherwise, $T$ rejects $A$ 's request. For these sessions, $T$ just randomly guesses the value of $b$, so the probability that $T$ wins the game is $\frac{1}{2}$.

To use $A$, after receiving first message $M_{1}=$ $\left(I D_{\text {new }}\right.$, sid,$\left.g^{a}\right), \quad T$ forms $M_{2}=\left(S I D\right.$, sid,$g^{b}$, $\operatorname{Sig}_{S K^{\prime}}\left(S I D, I D_{\text {new }}\right.$, sid $\left.\left., g^{a}, g^{b}\right)\right)$ by using $S K^{\prime}$ and sends it to $A$. If $A$ can successfully pass user authentication, s/he must be able to forge third message $M_{3}=\left(I D_{\text {new }}\right.$, sid, $\left.C T\right)$, where $C T=E_{P K}\left(C_{n e w}^{\prime}, I D_{n e w}, S I D\right.$, sid $\left., g^{a}, g^{b}\right)$. Now, $T$ requires to start the Game-PRF by choosing two distinct messages $y_{0}=H\left(I D_{n e w}\right)$ and $y_{1}=R_{1}$, and sends $\left(y_{0}, y_{1}\right)$ to the $P R F$ test query. The query responds $P R F_{x}\left(y_{b}\right)$ to $T$, then $T$ decrypts $C T$ to recover $C_{n e w}^{\prime}$ and checks whether the response is the same as $C_{n e w}^{\prime}$. If it is, then it outputs $b^{\prime}=0$ as the guessed result of $b$. Otherwise, it outputs $b^{\prime}=1$.

We now analyze the probability of game winning. We assume that $A$ forges user $U_{n e w}$, and passes user authentication successfully in polynomial time $\tau$, with non-negligible probability $\epsilon$, after asking $q_{R}$ times Register $\left(\prod, S_{j}\right), q_{E}$ times Execute $\left(U_{i}, S_{j}\right.$, sid $), q_{S}$ times send query in $q_{s}$ sessions. The formula of calculating probability $\operatorname{Pr}_{a d v}[P R F]$ of three different corrupting cases should be the same but with different $\epsilon$ because we do not care how $A$ can pass the user authentication. If $A$ does not select this special session, the probability of game wining without the help of $A$ is $\frac{1}{2}$. Otherwise, if $A$ indeed attacks this special session chose by 2 , then the probability is concerned as follows. The probability of $A$ pass authentication is $\epsilon$, so the probability that we win the Game-PRF is $\left(\epsilon \cdot 1+(1-\epsilon) \cdot \frac{1}{2}\right)$. Because if $A$ has passed authentication, then we have $100 \%$ probability to win the game. However, $A$ may fail with the probability of $(1-\epsilon)$, in this case, we have $\frac{1}{2}$ probability to win the game. Thus,

$$
\begin{aligned}
\operatorname{Pr}_{a d v}[P R F] & \frac{1}{q_{s}} \cdot\left(\epsilon \cdot 1+(1-\epsilon) \cdot \frac{1}{2}\right)+\frac{q_{s q_{s}}-1}{q_{s}} \cdot \frac{1}{2}-\frac{1}{2} \\
& =\frac{\epsilon+q_{s}}{2 q_{s}}-\frac{1}{2} \\
& =\frac{\epsilon}{2 q_{s}}
\end{aligned}
$$

It is clear that $\operatorname{Pr}_{a d v}[P R F]$ is non-negligible since $\epsilon$ is nonnegligible, and $T$ spends $\tau^{\prime}=\tau+\tau_{2}$ time to win games, where $\tau_{2}$ is the executing time of $T$ interaction with the test query. It is obvious that both $\tau$ and $\tau_{2}^{\prime}$ are polynomial times, thus, $\tau^{\prime}$ is also a polynomial time. Therefore, $T$ can win Game-PRF with non-negligible advantage $\operatorname{Pr}_{a d v}[P R F]$, and this contradicts assumption.

Lemma 2: (Secure Server Authentication) In proposed protocol $\Pi$, if the signature scheme is unforgeable against adaptive chosen message attack, and $\prod_{U_{i}, S_{j}}^{\text {sid }}$ has accepted, then for any PPT adversary $A$ in the adversary model, the 
probability of $N o-$ Matching $^{A, U_{i}}(k)$ is negligible.

Proof: This can be proved by contradiction as well. If $A$ has been accepted by $\prod_{U_{i}, S_{j}}^{s i d}$ with non-negligible probability of $N_{o}-$ Matching $^{A, U_{i}}(k)$, then we can construct a PPT machine $T$ which can win the Game of unforgeable against chosen message attack (Game-UFCMA) [47] by employing A.

In Game-UFCMA, there is a signature signing oracle $\prod_{\text {Sign }}$. A challenger who has $P K^{\prime}$ can make signing queries on messages, and can also verify the signature by using $P K^{\prime}$. To win the game, the challenger needs to output a fresh message $M_{\text {new }}$ with valid signature on it. Let $\operatorname{Pr}_{\text {win }}[S I G]$ be the probability of the advantage of game winning.

The basic idea is that to win Game-UFCMA, $T$ simulates an environment of our concrete protocol to convince adversary $A$ that this simulation is the real concrete protocol. In addition, $A$ should only have a negligible probability to know the truce, i.e. this is not a real protocol environment but a simulation. In such simulation, $T$ communicates with $A$ who has the ability to successfully forge server's signature in a session with session ID sid with a non-negligible probability. Then, $T$ will make use of $A$ 's ability to win Game-UFCMA with a non-negligible probability.

To use $A, T$ needs to simulate $A$ 's view as follows. In the simulation, $T$ answers all oracle queries made by $A$. To achieve this goal, $T$ needs to setup all parameters except signing key $S K^{\prime}$. In our concrete scheme, $A$ can ask following quires:

- Execute $\left(U_{i}, S_{j}\right.$, sid $)$ : In $\prod, \prod_{U_{i}, S_{j}}^{s i d}$ and $\prod_{S_{j}, U_{i}}^{s i d}$ generate and record all messages transmitted between $U_{i}$ and $S_{j}$, then send them to $A$.

- $\operatorname{Send}\left(U_{i}, S_{j}, \operatorname{sid}, M, m\right): A$ can send $M_{1}$ to $T$, then $T$ responds $M_{2}$ by asking the $\prod_{S i g n}$ of $\prod_{S_{j}, U_{i}}^{s i d}$. Upon receiving $M_{3}$ from $A, T$ sends the result of user authentication according to $M_{1}$ and $M_{3}$.

- $\operatorname{Send}\left(S_{j}, U_{i}\right.$, sid, $\left.M_{m^{\prime}}, m^{\prime}\right)$ : Upon receiving new session query $\operatorname{Send}\left(S_{j}, M_{\lambda}, \lambda\right), T$ asks $\prod_{U_{i}, S_{j}}^{s i d}$ to send first message $M_{1}$ to $A$. After receiving corresponding $M_{2}$, $T$ checks the signature, and forms $M_{3}$ if the signature is valid.

If $A$ can successfully pass server authentication with a non-negligible probability, there must exist a matching conversation between $A$ and $T$ who simulates user $U_{i}$ if the following happens. In the simulation, first, $T$ chooses message $M_{1}=\left(T, s i d, g^{a}\right)$, and sends it to $A$. If $A$ can successfully pass server authentication, then $A$ will form message $M_{2}=\left(S I D, \operatorname{sid}, g^{b}, \operatorname{Sig}_{S K^{\prime}}\left(S I D, T\right.\right.$, sid $\left.\left., g^{a}, g^{b}\right)\right)$ and send it to $T$.

To win the Game-UFCMA with $A$ 's help, $T$ sends $M=\left(S I D, T\right.$, sid $\left., g^{a}, g^{b}\right)$ together with the signature in $M_{2}$ to the test query. We assume that $A$ forges server $S$ and passes server authentication successfully in polynomial time $\tau$, with non-negligible probability $\epsilon$, asking $q_{E}$ times to $\operatorname{Execute}\left(U_{i}, S_{j}\right.$, sid $)$ and $q_{S}$ times to send a query, which contains $q_{s}$ times $\operatorname{Send}\left(S_{j}, U_{i}\right.$, sid, $\left.M_{m^{\prime}}, m^{\prime}\right)$. Let $\eta$ be the probability of $T$ winning Game-UFCMA when $A$ has failed to pass server authentication. The probability is analysed as follows. In $q_{s}$ times send query made by $A$, we choose one query to help us to answer the Game-UFCMA. The probability of $A$ pass sever authentication is $\epsilon$, so the probability of we win the Game-UFCMA is $(\epsilon \cdot 1+(1-\epsilon) \cdot \eta)$. Because that if $A$ has passed authentication, then we have $100 \%$ probability to win the game. On the other side, $A$ may also failed with the probability of $(1-\epsilon)$, in this case, we have the probability of $\eta$ to win the game. For the rest queries, the probability of game wining without the help of $A$ is $\eta$. Thus,

$$
\begin{aligned}
\operatorname{Pr}_{\text {win }}[S I G] & =\frac{1}{q_{s}} \cdot(\epsilon \cdot 1+(1-\epsilon) \cdot \eta)+\frac{q_{s}-1}{q_{s}} \cdot \eta \\
& =\frac{\epsilon+\eta \cdot\left(q_{s}-\epsilon\right)}{q_{s}}
\end{aligned}
$$

It is clear that $\operatorname{Pr}_{w i n}[S I G]$ is non-negligible since $\epsilon$ is non-negligible and $\eta$ is negligible. The time $T$ spent to win the games is $\tau^{\prime}=\tau+\tau_{3}$, where $t_{3}$ is the executing time of $T$ spends in GAME-UFCMA. $\tau^{\prime}$ is a polynomial time because both $\tau$ and $\tau_{3}^{\prime}$ are polynomial times. Therefore, we can construct PPT machine $T$ to win Game-UFCMA of the signature scheme, with non-negligible probability, and this is a contradiction.

Theorem 1: (Secure Three-Factor Mutual Authentication $(S T M A))$ In proposed protocol $\Pi$, if: (A) the $P R F$ is replaced by an ideal random function and $P K E$ scheme is secure against $C C A 2$ attack; (B) the signature scheme is unforgeable against chosen message attack; (C) at least one of $\prod_{U_{i}, S_{j}}^{s i d}$ and $\prod_{S_{j}, U_{i}}^{s i d}$ has accepted; then for any PPT adversary $A$ in the adversary model, the probabilities of both $N_{o}-$ Matching $^{A_{U_{i}}}(k)$ and No-Matching ${ }^{A_{S_{j}}}(k)$ are negligible.

Proof: Obviously, the first condition of Definition 2 holds because it is easy to verify that our concrete protocol is correct. In addition, by Lemma 1 and Lemma 2, the second and third conditions of Definition 2 also hold. Therefore, Theorem 1 holds.

Theorem 2: (Secure Three-Factor Authenticated Key Exchange (STAKE)) In proposed protocol $\prod$, if (A) the $P R F$ is replaced by an ideal random function and the $P K E$ scheme is secure against $C C A 2$ attack; (B) the signature scheme is unforgeable against adaptive chosen message attack; then for any PPT adversary $A$ in the adversary model, the advantage $A d v^{A}(k)$ of $A$ winning the game of $A K E P$ in a fresh session is negligible.

Proof: According to the Definition 3, STAKE needs to meet three conditions. The first condition is that protocol $\Pi$ is required to satisfies $S T M A$. This condition is achieved because Theorem 1 . The second condition is that for a fresh session in protocol $\prod$, if complete conversations are matched, then the same session key must be shared between these two communicating parties. This condition is achieved because that in our concrete scheme, the key exchange is the plain two-move Diffie-Hellman protocol [43], and this condition is a well-known property and it was proved. For the third condition, the advantage $A d v^{A}(k)=\left|\operatorname{Pr}\left[\operatorname{Gguess}^{A}(k)\right]-\frac{1}{2}\right|$ is non-negligible due to [43]. Thus, $\Pi$ is a secure three-factor authenticated key exchange protocol. 


\section{Privacy discussion}

The proposed framework preserves user privacy due to the following reasons. First, the server does not know any information about the user's biometric template since the user does not need to provide biometric templates to the server. Second, the data stored in $S C$ will not leak biometric information since $V$ contains a large amount of noise. Thus, the probability of successful recovering the biometric template is negligible due to [35]. Moreover, the helper data $H$ which is required in the fingerprint based fuzzy vault scheme are global features, and two very different fingerprint can have very similar helper data. So, $H$ also will not leak biometric characteristics [35].

\section{CONCLUSION}

The proposed framework can systematically and efficiently upgrade two-factor authentication schemes to three-factor authentication schemes. The derived scheme protects user's privacy, and enhances security. In addition, we made a case study by applying the framework on an existing two factor authentication scheme [41]. Our analysis, discussion, and formal proof show that the resulted three-factor protocol achieves higher security guarantee and preserves user privacy.

\section{ACKNOWLEDGMENTS}

The authors would like to thank The University of Birmingham for their financial support under the EPSRC grant $\mathrm{EP} / \mathrm{H} 005501 / 1$.

\section{REFERENCES}

[1] A. K. Jain, A. Ross, and S. Pankanti, "Biometrics: a tool for information security," IEEE Transactions on Information Forensics and Security, vol. 1, no. 2, pp. 125-143, 2006.

[2] J. Yu, G. Wang, and Y. Mu, "Provably secure single sign-on scheme in distributed systems and networks," in TrustCom, 2012, pp. 271-278.

[3] G. Wang, J. Yu, and Q. Xie, "Security analysis of a single sign-on mechanism for distributed computer networks," IEEE Trans. Industrial Informatics, vol. 9, no. 1, pp. 294-302, 2013.

[4] L. Lamport, "Password authentication with insecure communication," Commun. ACM, vol. 24, no. 11, pp. 770-772, Nov. 1981.

[5] C.-C. Chang and T.-C. Wu, "Remote password authentication with smart cards," IEE Proceedings-E Computers and Digital Techniques, vol. 138, no. 3, pp. 165 - 168, may 1991.

[6] A. Juels and M. Wattenberg, "A fuzzy commitment scheme," in ACM CCS, 1999, pp. 28-36.

[7] V. M. Jr. and Z. Ríha, "Toward reliable user authentication through biometrics," IEEE Security \& Privacy, vol. 1, no. 3, pp. 45-49, 2003.

[8] Z. Siddiqui, A.-H. Abdullah, M. K. Khan, and A. S. Alghamdi, "Smart environment as a service: Three factor cloud based user authentication for telecare medical information system," J. Medical Systems, vol. 38, no. 1, pp. 1-14, 2014.

[9] W.-H. Yang and S.-P. Shieh, "Password authentication schemes with smart cards," Computers \& Security, vol. 18, no. 8, pp. 727-733, 1999.

[10] L. Fan, J.-H. Li, and H.-W. Zhu, "An enhancement of timestamp-based password authentication scheme," Computers \& Security, vol. 21, no. 7, pp. 665-667, 2002.

[11] J.-J. Shena, C.-W. Linb, and M.-S. Hwang, "Security enhancement for the timestamp-based password authentication scheme using smart cards," Computers \& Security, vol. 22, no. 7, pp. 591-595, 2003.

[12] C.-C. Yanga, R.-C. Wang, and T.-Y. Chang, "An improvement of the yang-shieh password authentication schemes," Applied Mathematics and Computation, vol. 162, no. 3, pp. 1391-1396, 2005.

[13] E.-J. Yoon, W.-H. Kim, and K.-Y. Yoo, "Security enhancement for password authentication schemes with smart cards," in TrustBus, 2005, pp. 311-320.
[14] C.-K. Chan and L.-M. Cheng, "Cryptanalysis of timestamp-based password authentication scheme," Computers \& Security, vol. 21, no. 1, pp. 74-76, 2002.

[15] G. Wang and F. Bao, "Cryptanalysis of timestamp-based password authentication schemes using smart cards," in ICICS, 2006, pp. 399409.

[16] H.-S. Kim, S.-W. Lee, and K.-Y. Yoo, "Id-based password authentication scheme using smart cards and fingerprints," SIGOPS Oper. Syst. Rev., vol. 37, pp. 32-41, October 2003.

[17] M. Scott, "Cryptanalysis of an id-based password authentication scheme using smart cards and fingerprints," SIGOPS Oper. Syst. Rev., vol. 38, pp. 73-75, April 2004.

[18] U. Uludag, S. Pankanti, A. K. Jain, and S. Prabhakar, "Biometric cryptosystems: Issues and challenges," in Proceedings of the IEEE, 2004, pp. 948-960.

[19] A. K. Jain and D. Maltoni, Handbook of Fingerprint Recognition. Secaucus, NJ, USA: Springer-Verlag New York, Inc., 2003.

[20] A. Bhargav-Spantzel, A. C. Squicciarini, and E. Bertino, "Privacy preserving multi-factor authentication with biometrics," in Digital Identity Management, 2006, pp. 63-72.

[21] A. Bhargav-Spantzel, A. C. Squicciarini, S. K. Modi, M. Young, E. Bertino, and S. J. Elliott, "Privacy preserving multi-factor authentication with biometrics," Journal of Computer Security, vol. 15, no. 5, pp. 529-560, 2007.

[22] T. P. Pedersen, "Non-interactive and information-theoretic secure verifiable secret sharing," in CRYPTO, 1991, pp. 129-140.

[23] C.-I. Fan and Y.-H. Lin, "Provably secure remote truly three-factor authentication scheme with privacy protection on biometrics," IEEE Transactions on Information Forensics and Security, vol. 4, no. 4, pp. 933-945, 2009.

[24] X. Sun, H. Wang, J. Li, and Y. Zhang, "Satisfying privacy requirements before data anonymization," Comput. J., vol. 55, no. 4, pp. 422-437, 2012.

[25] L. A. Dunning and R. Kresman, "Privacy preserving data sharing with anonymous id assignment," IEEE Transactions on Information Forensics and Security, vol. 8, no. 2, pp. 402-413, 2013.

[26] C.-T. Li and M.-S. Hwang, "An efficient biometrics-based remote user authentication scheme using smart cards," J. Netw. Comput. Appl., vol. 33 , no. 1 , pp. 1-5, Jan. 2010.

[27] X. Li, J.-W. Niu, J. Ma, W.-D. Wang, and C.-L. Liu, "Cryptanalysis and improvement of a biometrics-based remote user authentication scheme using smart cards," Journal of Network and Computer Applications, vol. 34, no. 1, pp. 73-79, 2011.

[28] A. K. Das, "Cryptanalysis and further improvement of a biometric-based remote user authentication scheme using smart cards," International Journal of Network Security and Its Applications (IJNSA), vol. 3, no. 2, pp. 13-28, 2011.

[29] X. Huang, Y. Xiang, A. Chonka, J. Zhou, and R. H. Deng, "A generic framework for three-factor authentication: Preserving security and privacy in distributed systems," IEEE Transactions on Parallel and Distributed Systems, vol. 22, no. 8, pp. 1390 -1397, aug. 2011.

[30] Y. Dodis, L. Reyzin, and A. Smith, "Fuzzy extractors: How to generate strong keys from biometrics and other noisy data," in EUROCRYPT, 2004, pp. 523-540.

[31] Y. Wu and B. Qiu, "Transforming a pattern identifier into biometric key generators," in ICME, 2010, pp. 78-82.

[32] D. He, M. Ma, Y. Zhang, C. Chen, and J. Bu, "A strong user authentication scheme with smart cards for wireless communications," Computer Communications, vol. 34, no. 3, pp. 367-374, 2011.

[33] A. Nagar, K. Nandakumar, and A. K. Jain, "Securing fingerprint template: Fuzzy vault with minutiae descriptors," in 19th International Conference on Pattern Recognition, dec. 2008, pp. 1-4.

[34] T. C. Clancy, "Secure smartcard-based fingerprint authentication," in ACM Workshop on Biometrics: Methods and Applications, 2003, pp. 45-52.

[35] K. Nandakumar, A. K. Jain, and S. Pankanti, "Fingerprint-based fuzzy vault: Implementation and performance," IEEE Transactions on Information Forensics and Security, vol. 2, no. 4, pp. 744-757, 2007.

[36] U. Uludag and A. Jain, "Securing fingerprint template: fuzzy vault with helper data," in in Proceedings of CVPR Workshop on Privacy Research In Vision, 2006, p. 163.

[37] A. Juels and M. Sudan, "A fuzzy vault scheme," in International Symposium on Information Theory (ISIT). IEEE Press, July 2002, p. 408.

[38] J. Feng, "Combining minutiae descriptors for fingerprint matching," Pattern Recognition, vol. 41, no. 1, pp. 342-352, 2008. 
[39] B. T. Andrew and S. O. Thian, "Secure biometric template protection via randomized dynamic quantization transformation." IEEE International Symposium on Biometrics and Security Technologies, 2008.

[40] W. Sheng, G. Howells, M. C. Fairhurst, and F. Deravi, "Template-free biometric-key generation by means of fuzzy genetic clustering," IEEE Transactions on Information Forensics and Security, vol. 3, no. 2, pp. 183-191, 2008.

[41] G. Yang, D. S. Wong, H. Wang, and X. Deng, "Two-factor mutual authentication based on smart cards and passwords," J. Comput. Syst. Sci., vol. 74, pp. 1160-1172, November 2008.

[42] M. Bellare and P. Rogaway, "Entity authentication and key distribution," in CRYPTO, 1993, pp. 232-249.

[43] R. Canetti and H. Krawczyk, "Analysis of key-exchange protocols and their use for building secure channels," in EUROCRYPT, 2001, pp. 453 474.

[44] M. Bellare, D. Pointcheval, and P. Rogaway, "Authenticated key exchange secure against dictionary attacks," in EUROCRYPT, 2000, pp. $139-155$.

[45] J. Xu, W.-T. Zhu, and D. Feng, "An improved smart card based password authentication scheme with provable security," Computer Standards \& Interfaces, vol. 31, no. 4, pp. 723-728, 2009.

[46] J. Xu, W.-T. Zhu, and W. Jin, "A generic framework for constructing cross-realm c2c-paka protocols based on the smart card," Concurrency and Computation: Practice and Experience, vol. 23, no. 12, pp. 13861398, 2011.

[47] S. Goldwasser, S. Micali, and R. L. Rivest, "A digital signature scheme secure against adaptive chosen-message attacks," SIAM J. Comput., vol. 17 , no. 2, pp. 281-308, 1988.

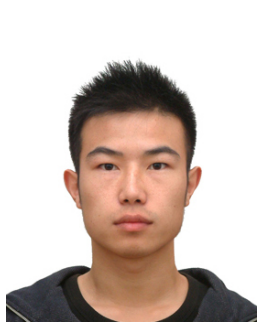

Jiangshan Yu received his MSc and MPhil degree in computer science (information security) respectively in 2011 and 2012, from the School of Computer Science and Software Engineering, University of Wollongong, Australia. He currently is a $\mathrm{PhD}$ candidate at University of Birmingham, U.K. The focus of his research has been on information security and privacy.

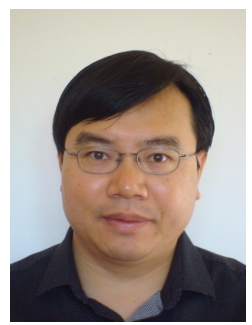

Guilin Wang received the $\mathrm{Ph} . \mathrm{D}$. degree in computer science from the Institute of Software, Chinese Academy of Sciences, Beijing, China, in 2001. He is currently a senior researcher with Huawei International Pte Ltd, Singapore. Before this, he was a Senior Lecturer in the University of Wollongong, Australia, a Lecturer in the University of Birmingham, U.K., a Research Scientist with the Institute for Infocomm Research, Singapore, and an Assistant Professor in the Chinese Academy of Sciences, Beijing, China. He has authored or coauthored more than 80 research publications in the areas of applied cryptography and telecommunication security. His main research interests include the analysis, design, and applications of digital signatures and security protocols. Dr. Wang has served as a program co-chair for six international security conferences, a committee member for more than 60 international conferences or workshops, and a reviewer for over 20 international journals.

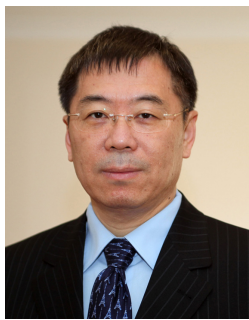

Yi Mu received his $\mathrm{PhD}$ from the Australian $\mathrm{Na}-$ tional University in 1994. He currently is professor, Head of School of Computer Science and Software Engineering and the co-director of Centre for Computer and Information Security Research at University of Wollongong, Australia. His current research interests include information security and cryptography. Yi Mu is the editor-in-chief of International Journal of Applied Cryptography and serves as associate editor for ten other international journals. He has published over 300 research papers. He is a senior member of the IEEE and a member of the IACR.

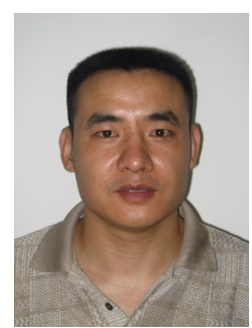

Wei Gao was born on Feb.2, 1978. He received B.Sc. degree from Ludong University (1996), M.S. degree from Guangzhou University (2003) and Ph.D. degree from Hunan University (2006). From 2007 he is an associate professor at Ludong University. His main research interests are appliced mathematics, cryptography and information security. 